\title{
Rapid molecular identification of fungal pathogens in corneal samples from suspected keratomycosis cases
}

Correspondence

Nicasio Mancini

nicasio.mancini@hsr.it

Received 21 March 2006

Accepted 8 August 2006

\author{
Nicasio Mancini, ${ }^{1,3}+$ Mario Perotti, ${ }^{1,3} \dagger$ Cristina M. Ossi, ${ }^{1}$ \\ Annalisa Cavallero, ${ }^{1}$ Stanislav Matuška, ${ }^{2}$ Giorgio Paganoni, ${ }^{2}$ \\ Roberto Burioni, ${ }^{1,3}$ Paolo Rama ${ }^{2}$ and Massimo Clementi ${ }^{1,3}$ \\ 1,2 Laboratorio di Microbiologia e Virologia ${ }^{1}$, and Centro Malattie della Cornea, Dipartimento di \\ Oftalmologia e Scienze della Visione ${ }^{2}$, IRCCS Istituto Scientifico San Raffaele, Milan, Italy \\ ${ }^{3}$ Università Vita-Salute San Raffaele, Facoltà di Medicina e Chirurgia, Milan, Italy
}

An increase in the incidence of fungal infections has highlighted the need for rapid and precise detection and identification methods in clinical mycology. This report describes the data obtained on corneal samples from 24 patients with suspected keratomycosis using a conventional cultural approach in parallel with PCR amplification and sequencing of the internal transcribed spacers (ITSs) of the rDNA regions. Using the cultural approach, seven samples (58.3\% of the 12 samples positive for an infectious pathogen) tested positive for a fungal aetiology, with final identification taking a mean time of more than 5 days. In two cases, diagnosis required 10 days. Using the ITS-based molecular approach, a direct diagnosis was obtained in only five of the seven fungus-positive cases (71.4\%) starting from the clinical samples, but identification was still possible in all seven cases within $24 \mathrm{~h}$ (by using $16 \mathrm{~h}$ cultures for the two remaining cases). Despite the less-than-optimal sensitivity when working directly on clinical samples, the obtained data indicate that the molecular strategy used in this study is a useful complement to the conventional diagnostic approaches used for keratomycosis and, in particular, allows precise and fast fungal identification, in response to the clinical requirements. Similar studies on larger panels of patients and on different clinical samples are required for further investigation of the clinical potential of ITS-based approaches in the diagnosis of mycotic infections.

\section{INTRODUCTION}

Keratitis is currently recognized as the most common ocular presentation of localized mycotic infections (Thomas, 2003). Indeed, the incidence of fungal infections of the eye has increased dramatically in the last few years, with some authors reporting up to $16-37 \%$ of keratitis cases related to a mycotic aetiology (Ferrer et al., 2001). The classical cultural approach is undoubtedly useful in mycotic keratitis, as well as in other fungal infections, but often takes more than a week for appropriate growth and final identification of the aetiological agent (Thomas, 2003). Additionally, conventional mycological identification can be elusive in some cases, especially when morphological characteristics are not easily differentiated (Guarro \& Gene, 2002; Mancini et al., 2005). Rapid and precise identification of the infectious agent is of epidemiological importance in medical mycology, as the emergence of unconventional fungal pathogens constitutively resistant to some antimycotic drugs and the

Abbreviation: ITS, internal transcribed spacer.

†These authors contributed equally to this work. broadening of the antimycotic panel have made it an important step in the choice of an effective therapy (Nucci, 2003; Pfaller \& Diekema, 2004). In this context, the development of a culture-independent, molecular-based approach could be of great diagnostic help in cases when a keratomycosis or any other severe fungal infection is suspected.

Several potentially discriminating, species-specific regions that are possibly useful for molecular diagnosis have been described in the genome of fungi (Biswas et al., 2003; Einsele et al., 1997; Hennequin et al., 1999; Makimura et al., 1994; Rakeman et al., 2005; Reiss et al., 2000). The internal transcribed spacer (ITS) 1 and ITS2 rDNA sequences offer several advantages in this perspective (Iwen et al., 2002; Leinberger et al., 2005; Pryce et al., 2003). The ITS regions are non-coding sequences interspaced among highly conserved fungal rDNA and have been shown to have a high heterogeneity among different fungal genera and species (Iwen et al., 2002; Pryce et al., 2003). Of note, sequence analysis of ITS regions has helped in the taxonomic positioning of some species that are not easily classifiable on 
the basis of a phenotypic approach (Graser et al., 2000; Guillot \& Gueho, 1995). However, despite its phylogenetic applications, the use of ITS regions in clinical diagnosis remains to be determined due to the lack of a complete molecular database allowing the systematic comparison of inter- and intraspecies variation in different isolates and, more importantly, due to the lack of studies investigating their direct diagnostic use on clinical samples. In order to evaluate the latter, we compared an ITS-based PCR approach with the classical cultural approach in a series of suspected cases of keratomycosis.

\section{METHODS}

Patients and specimens. Corneal scrapings were obtained from 24 patients with a clinical suspicion of keratomycosis. The patients were observed consecutively at the Center for Corneal Diseases of San Raffaele Hospital and Research Institute, Milan, Italy, between October 2004 and November 2005. At first presentation, all patients reported a history of suspected infectious keratitis that was refractive to topical antibiotics and presented signs of active infection at various stages of progression, as differently sized corneal ulcers with various grades of intraocular inflammation and various ranges of reduction in corneal thickness. No patient reported previous therapy with antimycotic agents. Each patient was then sampled either at presentation or following 1 day of wash-out. A written informed consent was signed by each patient allowing further sampling for molecular diagnosis.

Culture and staining. For the cultural approach, each corneal sample was inoculated directly on to a chocolate agar plate and a Sabouraud dextrose agar plate with chloramphenicol and gentamicin. The plates were incubated at $37^{\circ} \mathrm{C}\left(5 \% \mathrm{CO}_{2}\right)$ and $30^{\circ} \mathrm{C}$, respectively, for up to 10 days. When also suspected, an additional non-nutrient agar plate was used for culture of Acanthamoeba spp. $\left(37^{\circ} \mathrm{C}\right.$ for 10 days). For each sample, a Gram stain and, if necessary, a Calcofluor white stain were performed.

Molecular analysis. Each specimen was stirred directly into $200 \mu \mathrm{l}$ sterile saline and extracted using a QIAamp DNA mini kit (Qiagen) using a protocol adapted for extraction of DNA from fungal cells, as described elsewhere (Fredricks et al., 2005; Yamada et al., 2002). In brief, each sample was pre-incubated at $99^{\circ} \mathrm{C}$ for $20 \mathrm{~min}$ and then processed as suggested by the manufacturer. After addition of the cellular lysis buffer, the sample was incubated again at $99^{\circ} \mathrm{C}$ for $10 \mathrm{~min}$. The extracted DNA was amplified by PCR using a pair of universal fungal primers (V9D: 5'-TTAAGTCCCTGCCCTTTG TA3'; LS266: 5'-GCATTCCCAAACAACTCGACTC-3') encompassing highly conserved regions encoding fungal rDNA (Pryce et al., 2003). PCRs were performed in $0.2 \mathrm{ml}$ reaction tubes in a final volume of $50 \mu$ containing $1 \cdot 5 \mathrm{U}$ Platinum Taq DNA polymerase (Invitrogen),

Table 1. Results of Gram and Calcofluor white staining and species identification by culture and $P C R /$ sequencing

\begin{tabular}{|c|c|c|c|c|c|}
\hline Patient & Gram stain & Calcofluor white stain & $\begin{array}{c}\text { Culture for } \\
\text { bacteria and fungi* }\end{array}$ & $\begin{array}{c}\text { Culture for } \\
\text { Acanthamoeba }\end{array}$ & $\begin{array}{l}\text { ITS region } \\
\text { sequencing }\end{array}$ \\
\hline 1 & - & - & Scedosporium apiospermum (10 days) & - & Scedosporium apiospermum \\
\hline 3 & - & $\mathrm{ND}$ & - & ND & - \\
\hline 4 & - & - & Microsporum canis (10 days) & - & Microsporum canis \\
\hline 5 & - & Acanthamoeba cysts & - & + & - \\
\hline 7 & - & ND & - & ND & - \\
\hline 8 & - & ND & - & ND & - \\
\hline 9 & Hyphae & $\mathrm{ND}$ & Aspergillus flavus (4 days) & $\mathrm{ND}$ & Aspergillus flavus \\
\hline 10 & Yeasts & ND & Candida albicans (2 days) & ND & Candida albicans \\
\hline 11 & - & - & - & - & - \\
\hline 12 & - & - & - & + & - \\
\hline 16 & - & - & - & - & - \\
\hline 17 & - & ND & - & ND & - \\
\hline 18 & - & - & - & - & - \\
\hline 19 & Gram-positive cocci & ND & Staphylococcus epidermidis & ND & - \\
\hline 20 & - & ND & - & ND & - \\
\hline 21 & Hyphae & $\mathrm{ND}$ & Bipolaris spp. (6 days) & $\mathrm{ND}$ & Bipolaris spp. $\dagger$ \\
\hline 22 & - & - & Klebsiella pneumoniae & - & - \\
\hline 23 & - & - & Aspergillus flavus (4 days) & - & Aspergillus flavus $\dagger$ \\
\hline 24 & - & - & - & - & - \\
\hline
\end{tabular}

ND, Not done.

*The number of days required for definitive mycological identification is indicated in parentheses.

$\dagger$ Isolates were identified following extraction of DNA from cultured samples after $16 \mathrm{~h}$ of incubation. 
Isolate 1

S.apiospermum CBS

Isolate 1

S.apiospermum CBS

Isolate 1

S.apiospermum CBS

Isolate 1

S.apiospermum CBS

Isolate 1

S.apiospermum CBS

Isolate 2

Alternaria spp CBS

Asternaria

Alternaria spp CBS

Isolate 2

Alternaria spp CBS

Isolate 2

Alternaria spp CBS

Isolate 2

Alternaria spp CBS

Isolate 4

M. canis CBS

Isolate 4

M. canis CBS

Isolate 4

M. canis CBS

Isolate 4

M. canis CBS

Isolate 4

M. canis CBS

Isolate 4

M. canis CBS

Isolate 9

A.flavus ATCC

Isolate 9

A. flavus ATCC

Isolate 9

A.flavus ATCC

Isolate 9

A.flavus ATCC

Isolate 9

A.flavus ATCC

Isolate 10

C.albicans CBS

Isolate 10

c.albicans CBS

Isolate 10

c.albicans CBS

Isolate 10

C.albicans CBS

Isolate 21

Bipolaris spp CBS

Isolate 21

Bipolaris spp CBS

Isolate 21

Bipolaris spp CBS

Isolate 21

Bipolaris spp CBS

Isolate 21

Bipolaris spp CBS

Isolate 23

A. flavus ATCC

Isolate 23

A. flavus ATCC

Isolate 23

A. flavus ATCC

Isolate 23

A.flavus ATCC

Isolate 23

A.flavus ATCD
ACAGAGTTACTACTCCAAACCCATTGTGAACCTTACCTATGTTCTGTTGCCCTCGGCGGCGTCGGTCAGCGCCCC--TCTGAAAAGAGGACGATGCCCCTCCCGCCGGCAGCACCAAACT CTT-GAATTTTACAGCGGATTACAGTTCTGATTTGAAAACAAAAAACAAGTTAAAACTTTCAACAACGGATCTCTTGGTTCTGGCATCGATGA.AGAACGCAGCGAAATGCGATAAGTAA

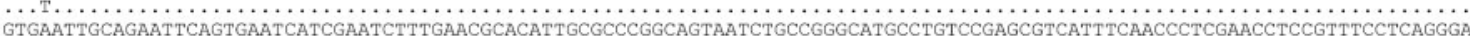

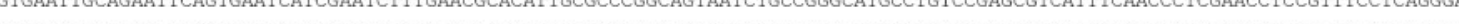
AGCCCAGGGTCGGTGTTGGGGCGCTACGGCGAGTCTTCGCGACCC-CCGTAGGCCCCGAAATACAGTGGCGGTCCCGCCGCGGTTGCCTTCTGCGTAGTAAGTCTCTTTTGCAAGCTCGC

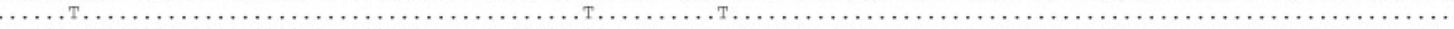

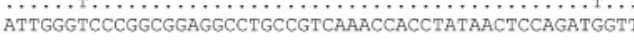

GAGGGATCATTACACAAATATGAAGGCGGGCTGGAACCTCTCGGGGTTACAGCCTTGCTGAATTATTCACCCTTGTCTTTTGCGTACTTCTTGTTTCCTTGGTGGGTTCGCCCACCACTA .

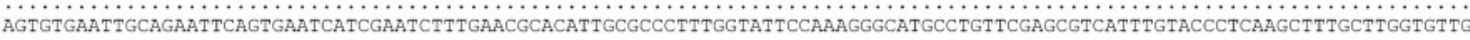

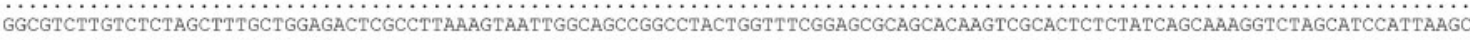

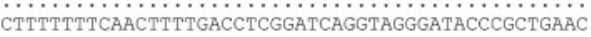

GAAGGATCATTAACGCGCAAGAGGTCGAAGTTGGCCCCCGAAGCTCTTCCGTCTCCCCCCCGGGCCTCCCGGGGAGGTTGCGGGCGGCGAGGGGTGCCTCCGGCCGCACGCCCATTCTTO

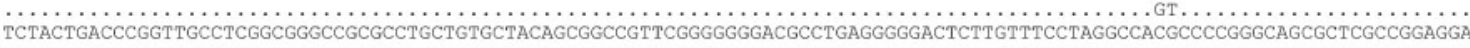

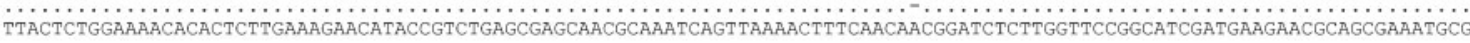

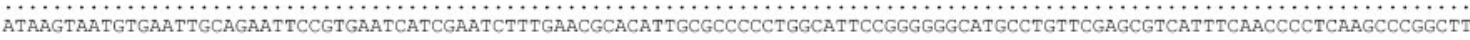

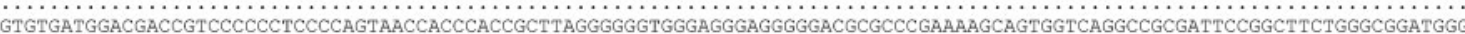

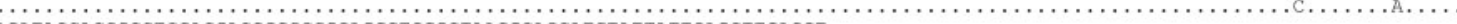
ACATACCACCGCCTCCAGGACCGGCCGGCAGGCTGGCCTAACGCACCATGTATTATTCAGGTTGACCT

GAAGGATCATTACCGAGTGTAGGGTTCCTAGCGAGCCCAACCTCCCAACCGTGTTTACTGTACCTTAGTTGCTTCGGCGGGTCCGCCATTCATGGCCGCCGGGGGCTCTCAGCCCCGGGC

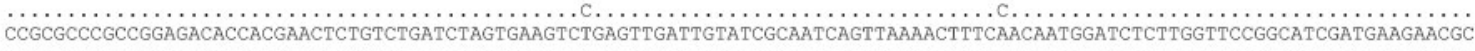
AGCGAAATGCGATAACTAGTGTGAATTGCAGAATTCCGTGAATCATCGAGTCTTTGAACGCACATTGCGCCCCCTGGTATTCCGGGGGGCATGCCTGTCCGAGCGTCATTGCTGCCCATC

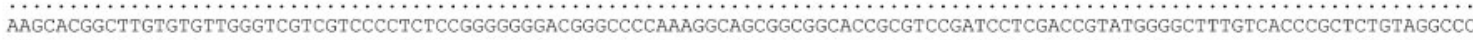

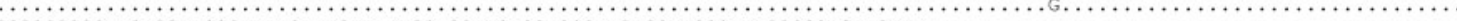
GGCCGGCGCTTGACGAACGCAAATCAATCTTTTICCAGGTTGACCTCGGATCAGGTAGGGATACCCGCTGAACTTAA

CTGATTTGCTTAATTGCACCACATGTGTTTTTCTTTGAAACAAACTTGCTTTGGCGGTAGGCCCAGCCTGCCGCCAGAG-TCTAAACTTACAACCAATTTTTTATCAACTTGTCACACCA

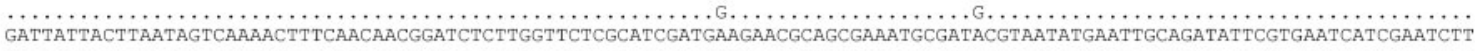

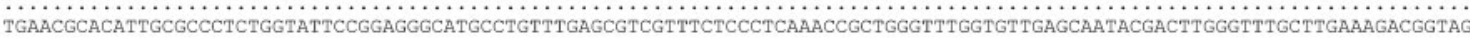
TGGTAAGGCGGGACCGCTTTGACAATGGCTTAGGTCTAACCAAAAACATTGCTTGCGGCGGTAGCGTCTACCACGTATATCTTCAAACT

GAGGGATCATTACACAATAAAATACGAAGGCCGTTCGCGGCTGGACTATTTCTTACCCTTGTCTTTTGCGCACTTGTTGTTTCCTGGGCGGGTTCGCTCGCCACCAGGACCACAATATAA

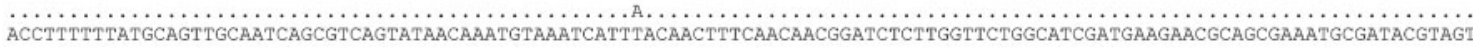
GTGAATTGCAGAATTCA GT GAATCATCGAATCTTTGAACGCACATTGCGCCCTTTGGTATTCCAAAGGGATGCCTGTTCGAGCGTCATTTGTACCCTCAAGCTTTGCTTGGTGTTGGGC

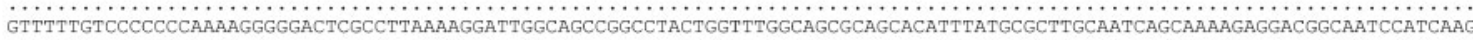

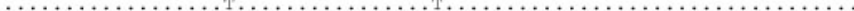

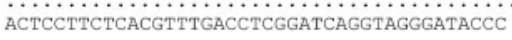

GAAGGATCATTACCGAGTGTAGGGTTCCTAGCGAGCCCAACCTCCCACCCGTGTTTACTGTACCTTAGTTGCTTCGGCGGGCCCGCCATTCATGGCCGCCGGGGGCTCTCAGCCCCGGGC

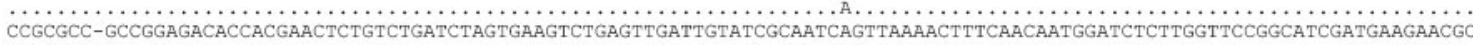

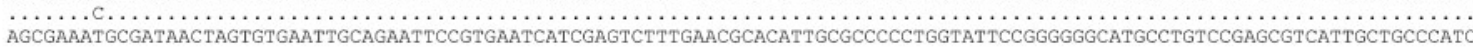

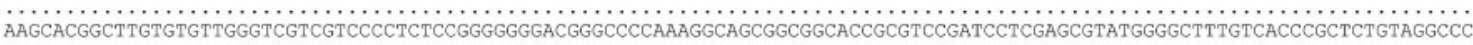

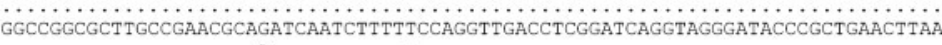

Fig. 1. ITS1-5.8S-ITS2 sequences from each corneal isolate were aligned with sequences from the BLAST database derived from the following reference strains: Scedosporium apiospermum (GenBank accession no. AJ888435); Alternaria spp. (GenBank accession no. AJ890440); Microsporum canis (GenBank accession no. MCA000619); Aspergillus flavus (GenBank accession no. AB008414); Candida albicans (GenBank accession no. AB018038); Bipolaris spp. (GenBank accession no. AY004776). At present, the absence of sequenced reference strains for different species of Alternaria and Bipolaris does not allow identification at the species level. In all cases percentage sequence similarity was higher than $98.5 \%$. 
$200 \mu \mathrm{M}$ each of dATP, dGTP and dCTP, $400 \mu \mathrm{M}$ dUTP (instead of dTTP), $20 \mathrm{mM}$ Tris/HCl (pH 8.4), $50 \mathrm{mM} \mathrm{MgCl}_{2}, 0 \cdot 4 \mu \mathrm{M}$ each primer and $1 \mathrm{U}$ uracil- $\mathrm{N}$-glycosylase. The amplification reaction included a hold at $50^{\circ} \mathrm{C}$ for 5 min to allow uracil- $N$-glycosylase activity and an additional hold at $95^{\circ} \mathrm{C}$ for $5 \mathrm{~min}$ for Taq activation, followed by 35 cycles at $95^{\circ} \mathrm{C}$ for $30 \mathrm{~s}, 62^{\circ} \mathrm{C}$ for $1 \mathrm{~min}$ and $72{ }^{\circ} \mathrm{C}$ for $2 \mathrm{~min}$, with a final extension step at $72{ }^{\circ} \mathrm{C}$ for $5 \mathrm{~min}$. The amplified product was visualized on agarose gels, purified and sequenced using two internal primers (ITS1: 5'-TCCGTAGGTGAACCTGCGG3'; ITS4: 5'-TCCTCCGCTTATTGATATGC-3'; Pryce et al., 2003; White et al., 1990) encompassing the ITS- $1,5 \cdot 8$ rRNA gene and ITS-2 regions. For each sample, a pair of primers amplifying the human $\beta$-globin gene was included as an extraction/amplification internal control. DNA sequences were analysed using the BLAST database and assigned to the reference isolate sequences with the highest bit score.

\section{RESULTS AND DISCUSSION}

Samples were processed in parallel using conventional culture methods and an ITS-based molecular approach and all results obtained with the two different approaches were included in the analysis (Table 1). Of the 24 samples, 12 $(50 \%)$ tested positive for an infectious pathogen by culture, with seven ( $58 \%$ of all positive samples) having a fungal aetiology. In four cases, evidence for a mycotic aetiology was obtained by direct staining (Gram and Calcofluor white stains), and identification by culture was possible only after a mean of more than 5 days of growth. In two cases, where less-common species were found (patients 1 and 4), diagnosis was not possible until 10 days of culture.

Using the ITS-based molecular approach, a directly amplified product was observed in five cases $(71 \cdot 4 \%$ of samples that were positive by culture), indicating a fungal aetiology; all other samples tested negative, including those in which other non-fungal pathogens were isolated. In the two additional cases (patients 21 and 23) that were positive for fungi by the classical approach, PCR amplification did not yield a positive result; however, in these cases, molecular identification was possible by extracting and amplifying DNA from tiny colonies obtained after only $16 \mathrm{~h}$ of culture, when conventional identification was not yet possible. All sequenced products were submitted to the BLAST database, taking into consideration for identification only complete ITS1-5.8S-ITS2 entries derived from reference isolates. In all cases, the clinical isolates showed a percentage sequence similarity of $>98.5 \%$ to the entry with the highest bit score, determining a BLAST search expect threshold of zero (Fig. 1).

In conclusion, the present report describes data obtained using two diagnostic strategies carried out in parallel on 24 cases of suspected keratomycosis: the conventional cultural approach and a PCR-based approach consisting of amplification and sequence analysis of the ITS1 and ITS2 regions of fungal DNA. In particular, the molecular strategy allowed a dramatic reduction in the time required for detection and identification of mycotic agents (with definitive species identification being obtained in less than 1 day when fungal DNA was directly amplified from the corneal sample), but in its present form it showed a lower sensitivity level than culture. Indeed, the described approach did not identify any fungal sequences in two out of seven samples that were positive for fungi by culture, indicating the need for improved sensitivity when working directly on clinical samples. Nonetheless, in the two discordant cases, definitive identification was possible from colonies before they could be assessed by classical identification. Of note, the present study indicates that the high specificity of the ITS regions, previously described only in studies performed on cultured isolates (Leinberger et al., 2005; Pryce et al., 2003), makes them suitable for identification of fungal pathogens directly from clinical samples; indeed, no false-positive reactions related to cross-amplification of human DNA or DNA derived from other sources (shown by the absence of amplified product in the five samples that were positive for non-fungal pathogens) were observed. These features, along with their potential use on automated supports, make ITS sequences valuable targets for setting up molecular approaches aimed at direct and rapid diagnosis of fungal pathogens at the species level, and could also be applied to non-ocular samples that are heavily contaminated by resident flora, as well as to samples from sterile sites. In conclusion, the data presented here support the notion that ITS-based molecular methods may be an important complement to conventional mycological detection by culture and may constitute an unequivocal and rapid means of fungal identification, which is becoming increasingly important in clinical mycology.

\section{REFERENCES}

Biswas, S. K., Wang, L., Yokoyama, K. \& Nishimura, K. (2003). Molecular analysis of Cryptococcus neoformans mitochondrial cytochrome $b$ gene sequences. J Clin Microbiol 41, 5572-5576.

Einsele, H., Hebart, H., Roller, G. \& 8 other authors (1997). Detection and identification of fungal pathogens in blood by using molecular probes. J Clin Microbiol 35, 1353-1360.

Ferrer, C., Colom, F., Frasés, S., Mulet, E., Abad, J. L. \& Alió, J. L. (2001). Detection and identification of fungal pathogens by PCR and by ITS2 and $5 \cdot 8 \mathrm{~S}$ ribosomal DNA typing in ocular infections. J Clin Microbiol 39, 2873-2879.

Fredricks, D. N., Smith, C. \& Meier, A. (2005). Comparison of six DNA extraction methods for recovery of fungal DNA as assessed by quantitative PCR. J Clin Microbiol 43, 5122-5128.

Gräser, Y., Kuijpers, A. F. A., El Fari, M., Presber, W. \& de Hoog, G. S. (2000). Molecular and conventional taxonomy of the Microsporum canis complex. Med Mycol 38, 143-153.

Guarro, J. \& Gené, J. (2002). Acrophialophora fusispora misidentified as Scedosporium prolificans. J Clin Microbiol 40, 3544-3545.

Guillot, J. \& Gueho, E. (1995). The diversity of Malassezia yeasts confirmed by rRNA sequence and nuclear DNA comparisons. Antonie van Leeuwenhoek 67, 297-314.

Hennequin, C., Abachin, E., Symoens, F., Lavarde, V., Reboux, G., Nolard, N. \& Berche, P. (1999). Identification of Fusarium species involved in human infections by $28 \mathrm{~S}$ rRNA gene sequencing. J Clin Microbiol 37, 3586-3589. 
Iwen, P. C., Hinrichs, S. H. \& Rupp, M. E. (2002). Utilization of the internal transcribed spacer regions as molecular targets to detect and identify human fungal pathogens. Med Mycol 40, 87-109.

Leinberger, D. M., Schumacher, U., Autenrieth, I. B. \& Bachmann, T. T. (2005). Development of a DNA microarray for detection and identification of fungal pathogens involved in invasive mycoses. J Clin Microbiol 43, 4943-4953.

Makimura, K., Murayama, S. Y. \& Yamaguchi, H. (1994). Detection of a wide range of medically important fungi by the polymerase chain reaction. J Med Microbiol 40, 358-364.

Mancini, N., Ossi, C. M., Perotti, M. \& 9 other authors (2005). Direct sequencing of Scedosporium apiospermum DNA in the diagnosis of a case of keratitis. J Med Microbiol 54, 897-900.

Nucci, M. (2003). Emerging moulds: Fusarium, Scedosporium and Zygomycetes in transplant recipients. Curr Opin Infect Dis 16, 607-612.

Pfaller, M. A. \& Diekema, D. J. (2004). Rare and emerging opportunistic fungal pathogens: concern for resistance beyond Candida albicans and Aspergillus fumigatus. J Clin Microbiol 42, 4419-4431.
Pryce, T. M., Palladino, S., Kay, I. D. \& Coombs, G. W. (2003). Rapid identification of fungi by sequencing the its1 and its2 regions using an automated capillary electrophoresis system. Med Mycol 41, 369-381.

Rakeman, J. L., Bui, U., Lafe, K., Chen, Y.-C., Honeycutt, R. J. \& Cookson, B. T. (2005). Multilocus DNA sequence comparisons rapidly identify pathogenic molds. J Clin Microbiol 43, 3324-3333.

Reiss, E., Obayashi, T., Orle, K., Yoshida, M. \& Zancopé-Oliveira, R. M. (2000). Non-culture based diagnostic tests for mycotic infections. Med Mycol 38 (Suppl. 1), 147-159.

Thomas, P. A. (2003). Current perspectives on ophthalmic mycoses. Clin Microbiol Rev 16, 730-797.

White, T., Burns, T., Lee, S. \& Taylor, J. (1990). Amplification and direct sequencing of fungal ribosomal RNA genes for phylogenetics. In PCR Protocols, pp. 315-322. Edited by M. A. Innis, D. H. Gelfand, J. J. Sninsky \& T. J. White. San Diego: Academic Press.

Yamada, Y., Makimura, K., Merhendi, H., Ueda, K., Nishiyama, Y., Yamaguchi, H. \& Osumi, M. (2002). Comparison of different methods for extraction of mitochondrial DNA from human pathogenic yeasts. Jpn J Infect Dis 55, 122-125. 